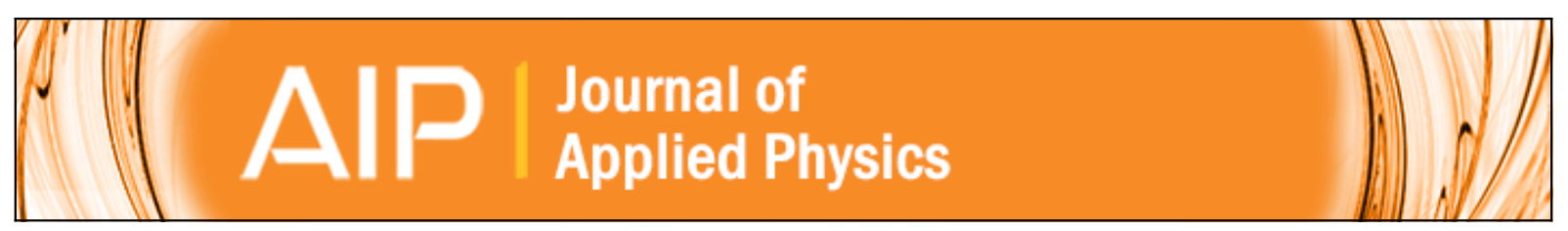

\title{
Perpendicular-bias-field-dependent vortex-gyration eigenfrequency
}

Myoung-Woo Yoo, Ki-Suk Lee, Dong-Soo Han, and Sang-Koog Kim

Citation: Journal of Applied Physics 109, 063903 (2011); doi: 10.1063/1.3563561

View online: http://dx.doi.org/10.1063/1.3563561

View Table of Contents: http://scitation.aip.org/content/aip/journal/jap/109/6?ver=pdfcov

Published by the AIP Publishing

\section{Articles you may be interested in}

Micromagnetic simulations of magnetic normal modes in elliptical nanomagnets with a vortex state

Appl. Phys. Lett. 103, 252404 (2013); 10.1063/1.4850537

Magnetic vortex echoes

J. Appl. Phys. 112, 113911 (2012); 10.1063/1.4768446

Radial-spin-wave-mode-assisted vortex-core magnetization reversals

Appl. Phys. Lett. 100, 172413 (2012); 10.1063/1.4705690

Sub-nanosecond switching of vortex cores using a resonant perpendicular magnetic field Appl. Phys. Lett. 100, 082402 (2012); 10.1063/1.3687909

Limits for the vortex state spin torque oscillator in magnetic nanopillars: Micromagnetic simulations for a thin free layer

J. Appl. Phys. 108, 123914 (2010); 10.1063/1.3524222

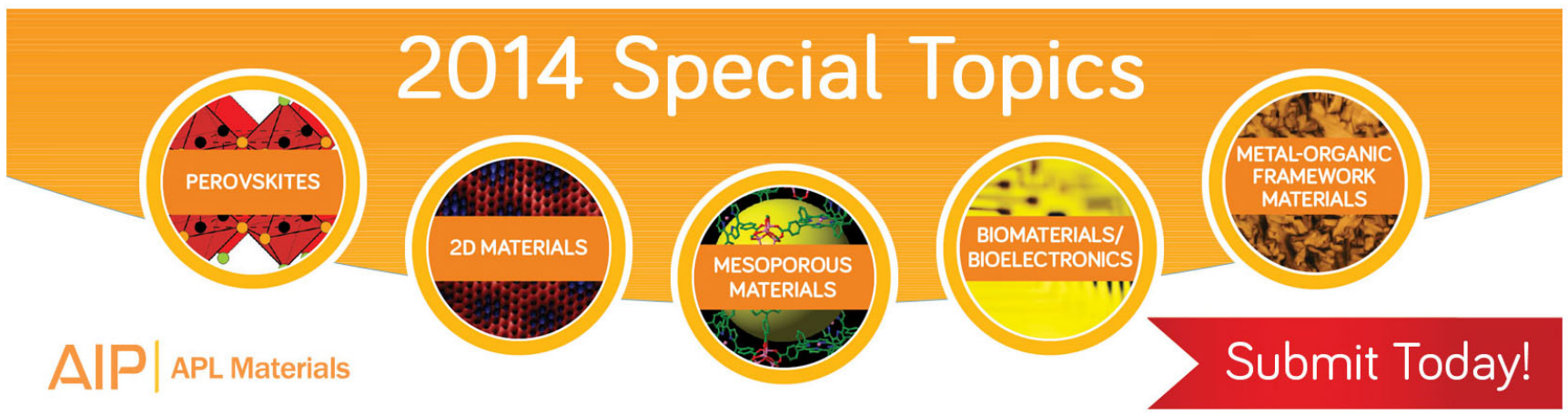




\title{
Perpendicular-bias-field-dependent vortex-gyration eigenfrequency
}

\author{
Myoung-Woo Yoo, Ki-Suk Lee, Dong-Soo Han, and Sang-Koog Kim ${ }^{\text {a) }}$ \\ Research Center for Spin Dynamics \& Spin-Wave Devices, Nanospinics Laboratory, Research Institute \\ of Advanced Materials, Department of Materials Science and Engineering, Seoul National University, \\ Seoul 151-744, South Korea
}

(Received 13 January 2011; accepted 22 January 2011; published online 17 March 2011)

\begin{abstract}
We found that the angular frequency $\omega_{0}$ of vortex-core gyrations is controllable by the application of static perpendicular bias fields $H_{\mathrm{p}}$ as studied by micromagnetic simulations and Thiele's-approach-based quantitative interpretation. The observed linear dependence of $\omega_{0}$ on $H_{\mathrm{p}}$ could be explained in terms of the dynamic variables of the vortex, the gyrovector constant $G$, and the potential stiffness constant $\kappa$, for cases of negligible damping. Here we calculated the values of $G$ and $\kappa$ as a function of $H_{\mathrm{p}}$ directly from the simulation numerical data using Thiele's equivalent force equations, providing a more correct understanding of the remarkable change of $\omega_{0}$ with $H_{\mathrm{p}}$. This micromagnetic-simulation-based quantitative analysis is a straightforward, accurate, and effective means of understanding vortex dynamics in nanoscale magnetic elements. (C) 2011 American Institute of Physics.

[doi:10.1063/1.3563561]
\end{abstract}

\section{INTRODUCTION}

Magnetic vortex configurations have received a great deal of attention, not only because of the vortex structure's unique fourfold ground states in magnetic nanodots of micrometer size or smaller ${ }^{1,2}$ but also due to its nontrivial vortex-core reversal dynamics. ${ }^{3-7}$ The fourfold vortex states are represented by combinations of either counter-clockwise $(\mathrm{CCW})$ or clockwise $(\mathrm{CW})$ in-plane curling magnetization and either up- or downward out-of-plane magnetization at the core. ${ }^{1}$ Owing not only to the binary representation of the core orientation ${ }^{8}$ but also to the full degrees of freedom of the fourfold ground states, ${ }^{9}$ such a stable vortex structure has been considered as a candidate for potential information storage applications. ${ }^{10}$ Furthermore, the existence of the vortex core, combined with the restoring force associated with nanodots' finite lateral size, gives rise to in-plane rotating motions of the core around its equilibrium center position at the characteristic angular eigenfrequency $\omega_{0}$ in either the $\mathrm{CCW}$ or $\mathrm{CW}$ angular rotation direction, so-named the gyrotropic mode. ${ }^{11}$ Such persistent vortex oscillations, with their high power output and narrow line widths, have been studied intensively recently for their promising application to microwave emission sources. ${ }^{12}$

It is well known that the rotation sense of the gyrotropic mode is determined by the core magnetization direction ${ }^{11}$ and that the value of $\omega_{0}$ depends on the dot dimensions, that is, thickness-to-radius $(L / R)$ ratio for the given material. ${ }^{13} \omega_{0}$ typically falls within the $50-600 \mathrm{MHz}$ range for $L / R$ ratios between 0.01 and $0.12 .^{13}$ The $\omega_{0}$, then, is not controllable for a given dot. Finding a way to manipulate it with external parameters such as fields, meanwhile, is a challenge. Choi et $a l .{ }^{14}$ reported that the eigenfrequency can be modified by Oersted fields produced by an out-of-plane dc current, specifically by varying the potential energy of the dots. Very

\footnotetext{
a) Author to whom correspondence should be addressed. Electronic mail: sangkoog@snu.ac.kr.
}

recently, Loubens et al. ${ }^{15}$ reported an experimental observation of the dependence of $\omega_{0}$ on the strength of a static magnetic field $H_{\mathrm{p}}$ applied perpendicularly to the nanodot plane. However, the underlying physics have yet to be quantitatively unraveled. In this paper, we report, on the basis of micromagnetic simulations, that the value of $\omega_{0}$ is controllably variable linearly with $H_{\mathrm{p}}$ in its sufficiently wide but limited range. Furthermore, this work provides a more correct understanding of the linear dependence of $\omega_{0}$ on $H_{\mathrm{p}}$ as determined by direct calculation of the $H_{\mathrm{p}}$ dependences of the dynamic variables of the vortex through Thiele's equivalent force equation, ${ }^{16}$ using the numerical simulation data.

\section{MODELING AND MICROMAGNETIC SIMULATION}

In the present micromagnetic simulations, we used the OOMMF code ${ }^{17}$ implementing the Landau-Lifshitz-Gilbert (LLG) equation ${ }^{18}$ of motion. We chose, for the model system, a permalloy ( $\mathrm{Py}: \mathrm{Ni}_{80} \mathrm{Fe}_{20}$ ) nanodisk of $300 \mathrm{~nm}$ radius and $30 \mathrm{~nm}$ thickness [Fig. 1(a)]. The typical Py material parameters (saturation magnetization $M_{\mathrm{s}}=860 \mathrm{emu} / \mathrm{cm}^{3}$, exchange stiffness $A_{\mathrm{ex}}=1.3 \times 10^{-6} \mathrm{erg} / \mathrm{cm}$, with zero magnetocrystalline anisotropy) were employed. The size of the individual cells was $2 \times 2 \times 30 \mathrm{~nm}^{3}$, and the Gilbert damping constant was $\alpha=0.01$. Static magnetic fields were applied perpendicularly to the dot plane, and the strength varied from $H_{\mathrm{p}}=-3$ to $3 \mathrm{kOe}$ at intervals of $1 \mathrm{kOe} .{ }^{19}$ The negative (positive) sign corresponds to the antiparallel (parallel) orientation of the core magnetization to the field direction.

\section{RESULTS AND DISCUSSION}

To examine how $\omega_{0}$ varies with $H_{\mathrm{p}}$, we used the following typical approach: first, we shifted the core from the center position by application of a static field of $H_{y}=100$ Oe in the $+y$ direction; then, during free relaxation after the inplane field was turned off, the averaged oscillations of the $m_{x}=M_{x} / M_{\mathrm{s}}$ component $\left(\left\langle m_{x}\right\rangle\right)$ were calculated as a function 

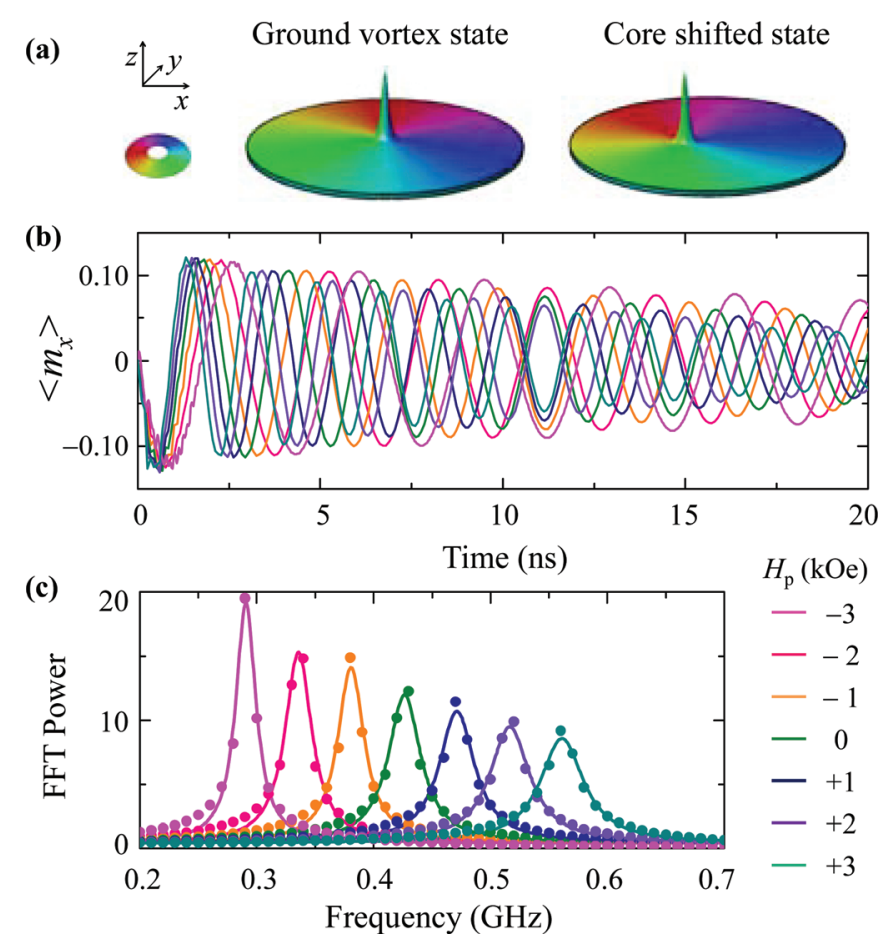

FIG. 1. (Color online) (a) Ground vortex state (left) and core-shifted state (right) with upward core magnetization and counter-clockwise (CCW) in-plane curling magnetization in Py disk of diameter $2 R=600 \mathrm{~nm}$ and thickness $L=30 \mathrm{~nm}$. The colors and heights represent the local in- and outof-plane magnetization components, respectively. (b) Average $m_{x}$ component $\left\langle m_{x}\right\rangle$ over whole disk vs time under perpendicular bias fields of indicated strength $H_{\mathrm{p}}$, during relaxation process after vortex core is shifted. (c) FFT power spectra for different $H_{\mathrm{p}}$ values, obtained from FFTs of $\left\langle m_{x}\right\rangle$ oscillations over $t=0-100 \mathrm{~ns}$ range.

of time for different $H_{\mathrm{p}}$ values [Fig. 1(b)]. Through fast Fourier transforms (FFTs) of the $\left\langle m_{x}\right\rangle$ oscillations, we plotted FFT powers in the frequency domain as a function of $H_{\mathrm{p}}$, as shown in Fig. 1(c). The individual dominant frequency peaks represent $\omega_{0} / 2 \pi$ for a given $H_{\mathrm{p}}$. The value of $\omega_{0} / 2 \pi$ at $H_{\mathrm{p}}=0$ is about $430 \mathrm{MHz}$, which increases monotonically to $560 \mathrm{MHz}$ at $H_{\mathrm{p}}=+3 \mathrm{kOe}$ and which decreases to $290 \mathrm{MHz}$ at $H_{\mathrm{p}}=-3 \mathrm{kOe}$. The $\omega_{0}$ variation with $H_{\mathrm{p}}$ (closed circles), as can be seen in Fig. 2, shows a linear dependence.

To understand such a linear response, we start with Thiele's analytical approach. ${ }^{16}$ Assuming a steady-state motion with core velocity $\mathbf{v}$, the governing equation of the gyrotropic mode is given as $-\mathbf{G} \times \mathbf{v}-\hat{D} \mathbf{v}+\partial W(\mathbf{X}) /$ $\partial \mathbf{X}=0$, where $\mathbf{G}=-p G \hat{\mathbf{z}}$ is the gyrovector with its constant $G>0$ and the core polarization $p$, and $\hat{D}=D \hat{I}$ is the damping tensor with the identity matrix $\hat{I}$ and its constant $D<0$. The potential energy function is given as $W(\mathbf{X})=W(0)+1 / 2 \kappa|\mathbf{X}|^{2}$ with the vortex-core position vector $\mathbf{X}$ for a linear regime. ${ }^{11}$ The first term is the potential energy for $\mathbf{X}=0$, and the second, the potential energy of the intrinsic stiffness coefficient $\kappa$ for displaced core position $\mathbf{X}$, as dominated by exchange and magnetostatic energies. Solving this governing equation, the characteristic angular eigenfrequency is given as $\omega_{D}=\kappa G /\left(G^{2}+D^{2}\right)$ with a certain damping $D .{ }^{20}$ For cases of negligible damping, that is, $G \gg|D|, \omega_{D}$ becomes approximately $\omega_{0}=\kappa / G .{ }^{11}$ Based on a two-vortex side surface charge free model, ${ }^{11,13,21} G$ and $\kappa$ can be expressed explicitly as $G=2 \pi L M_{\mathrm{s}} / \gamma$ with the gyro-

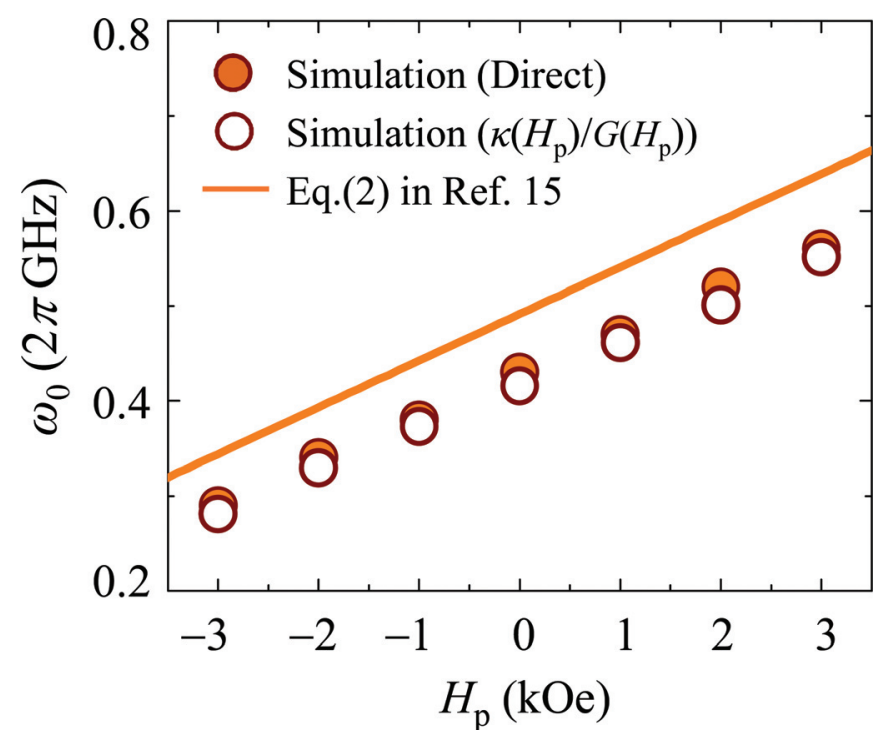

FIG. 2. (Color online) $H_{\mathrm{p}}$ dependence of $\omega_{0}$ (closed circles), obtained from FFT power spectra [Fig. 1(c)], compared with results for $\kappa\left(H_{\mathrm{p}}\right) / G\left(H_{\mathrm{p}}\right)$ (open circles) obtained from $H_{\mathrm{p}}$-dependent $G$ and $\kappa$ values (see Fig. 3), also obtained directly from simulation data. The solid line indicates the result of Eq. (2) reported in Ref. 15.

magnetic ratio $\gamma$, and $\kappa=\frac{40}{9} \pi M_{\mathrm{s}}^{2} L^{2} / R$ when $L / R \ll 1$. Accordingly, $\omega_{0}$ is explicitly given as $\omega_{0}=\frac{20}{9} M_{\mathrm{s}} \gamma L / R$ (Ref. 13 ) and hence is determined only by the $L / R$ aspect ratio for a given material. As such, the $H_{\mathrm{p}}$ dependence of $\omega_{0}$ shown in Fig. 2 (closed circles) cannot be understood. Recently Loubens et al. ${ }^{15}$ reported an experimental result of the linear response, describing the result as an explicit expression of $\omega_{0}\left(H_{\mathrm{p}}\right)=\omega_{0}(0)\left[1+p\left(H_{\mathrm{p}} / H_{\mathrm{s}}\right)\right]$, where $H_{\mathrm{s}}$ is the field to saturate the dot along the normal (see solid line in Fig. 2). Our simulation results and the analytical expression were found to be in general agreement but with $\sim 14 \%$ difference. The analytical form was estimated from the relation of $\omega_{0}=\kappa / G$ (Ref. 11) for the case $H_{\mathrm{p}}=0$ by employing the estimated analytical forms $G\left(H_{\mathrm{p}}\right)=G(0)\left[1-p\left(H_{\mathrm{p}} / H_{\mathrm{s}}\right)\right]$ and $\kappa\left(H_{\mathrm{p}}\right)$ $=\kappa(0)\left[1-\left(H_{\mathrm{p}} / H_{\mathrm{s}}\right)^{2}\right] \cdot{ }^{15}$ However, such explicit expressions cannot properly be derived analytically due to the fact that because the application of $H_{\mathrm{p}}$ modifies the ground magnetization state of a vortex, the two-vortex side surface charge free model, which was used to derive analytical equations of $G$ and $\kappa$ at $H_{\mathrm{p}}=0$, cannot be applicable to the cases of application of perpendicular fields.

For a deeper or more correct understanding, the variations of $G$ and $\kappa$ with $H_{\mathrm{p}}$ should be calculated from the results of simulations in which variations of the vortex ground state with $H_{\mathrm{p}}$ are accounted for. Our main idea is to use the equivalent force balance equation $\mathbf{F}^{G}+\mathbf{F}^{D}+\mathbf{F}^{W}=0$, where each term corresponds to the gyrotropic force, the damping force, and the restoring force due to the potential energy $W$, respectively, as indicated by the symbols in the superscript. The values of $G$ and $D$ can be extracted directly from those force terms $\mathbf{F}^{G}=p G \hat{\mathbf{z}} \times \mathbf{v}$ and $\mathbf{F}^{D}=\hat{D} \cdot \mathbf{v}=D \mathbf{v}$, without any assumption, when the values of $\mathbf{v}, \mathbf{F}^{G}$, and $\mathbf{F}^{D}$ are known. The force terms can be calculated from the simulation numerical data by $\mathbf{F}^{G}=\sum \mathbf{f}_{i}^{G}$ and $\mathbf{F}^{D}=\sum \mathbf{f}_{i}^{D}$ with $\mathbf{f}_{i}^{G}=\left(f_{i, x}^{G}, f_{i, y}^{G}\right)$, $\mathbf{f}_{i}^{D}=\left(f_{i, x}^{D}, f_{i, y}^{D}\right)$, where each term for the $i$ th cell is given as 


$$
\begin{gathered}
f_{i, x}^{G}=-\left(1 / \gamma M_{\mathrm{s}}^{2}\right)(\mathbf{M} \times d \mathbf{M} / d t) \cdot \partial \mathbf{M} / \partial x, \\
f_{i, y}^{G}=-\left(1 / \gamma M_{\mathrm{s}}^{2}\right)(\mathbf{M} \times d \mathbf{M} / d t) \cdot \partial \mathbf{M} / \partial y, \\
f_{i, x}^{D}=-\left(\alpha / \gamma M_{\mathrm{s}}\right)(d \mathbf{M} / d t) \cdot \partial \mathbf{M} / \partial x, \\
f_{i, y}^{D}=-\left(\alpha / \gamma M_{\mathrm{s}}\right)(d \mathbf{M} / d t) \cdot \partial \mathbf{M} / \partial y .
\end{gathered}
$$

The calculation results (open circles) for $G$ as functions of $H_{\mathrm{p}}$, using the approach explained in the preceding text, are shown in Fig. 3(a). The slope of $\Delta G / \Delta H_{\mathrm{p}}$, plotted in Fig. 3(a), is about $-9.8 \times 10^{-11} \mathrm{erg} \cdot \mathrm{s} /\left(\mathrm{cm}^{2} \cdot \mathrm{kOe}\right)$. The negative sign indicates that $G$ decreases with increasing $H_{\mathrm{p}}$. This is consistent with the fact that $G$ is determined by the relative magnitude of the out-of-plane magnetization of the core with respect to those around it. ${ }^{16}$ This variation is in good agreement (within a $6 \%$ difference) with the explicit expression (solid line) $G\left(H_{\mathrm{p}}\right)=G(0)\left[1-p\left(H_{\mathrm{p}} / H_{\mathrm{s}}\right)\right]$ with $G(0)$ $=2 \pi L M_{s} / \gamma$ (Ref. 11) for $\gamma=2 \pi \times 2.8 \mathrm{MHz} / \mathrm{Oe}, L=30 \mathrm{~nm}$, and $H_{\mathrm{s}}=10 \mathrm{kOe}$, as obtained for our nanodot dimensions.

The calculation results of $D$ are shown in Fig. 3(b). $D$ increases with increasing $H_{\mathrm{p}}$ but deviates slightly from the linear response in the negative $H_{\mathrm{p}}$ region. $D=-2.1402 \times 10^{-11}$ $\mathrm{erg} \cdot \mathrm{s} / \mathrm{cm}^{2}$ at $H_{\mathrm{p}}=0$ is close to the value $-2.3016 \times 10^{-11}$ $\mathrm{erg} \cdot \mathrm{s} / \mathrm{cm}^{2}$ obtained from the analytic equation $D=-\alpha \pi M_{\mathrm{s}} L$ $\left[2+\ln \left(R / R_{\mathrm{c}}\right)\right] / \gamma$ (Ref. 22) for $R=300 \mathrm{~nm}$ and $L=30 \mathrm{~nm}$ and assuming the vortex-core radius $R_{\mathrm{c}}=15 \mathrm{~nm}$. This equation was derived for the case of $H_{\mathrm{p}}=0$ based on the model proposed by Usov et al. ${ }^{23}$ The $G \gg|D|$ results plotted in Fig. 3 indicate that the damping term only negligibly contributes to the eigenfrequency.

Next, the $H_{\mathrm{p}}$ dependence of the $\kappa$ value was numerically calculated from the simulation data using $W(\mathbf{X})=W(0)$ $+1 / 2 \kappa|\mathbf{X}|^{2}$. To calculate the total potential energy $W$ versus
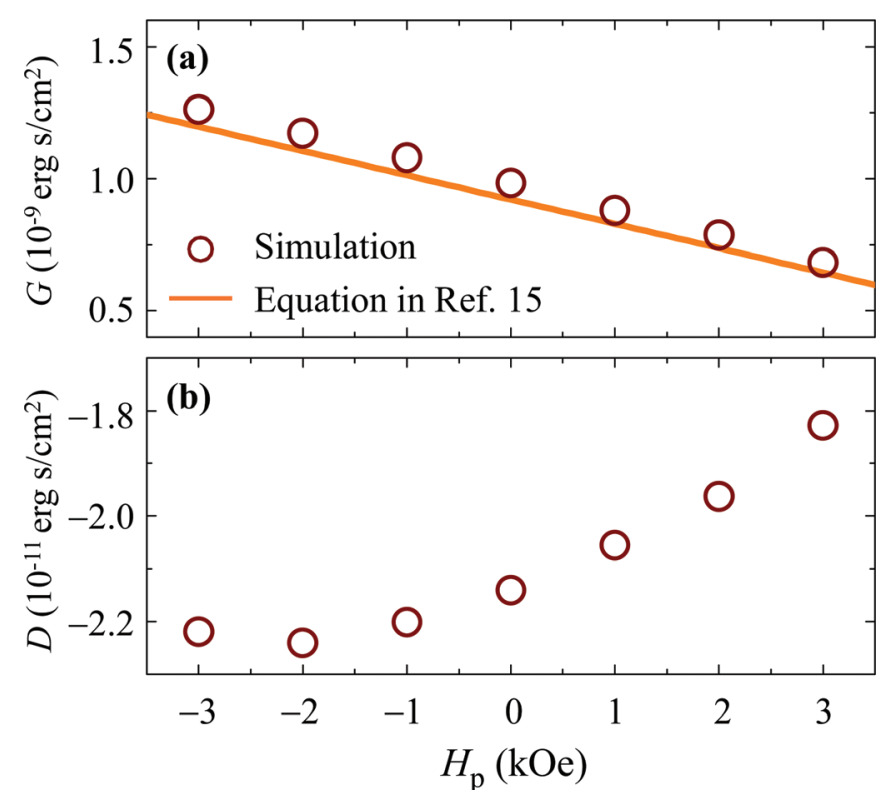

FIG. 3. (Color online) Dependences of dynamic variables $G$ and $D$ on $H_{\mathrm{p}}$. The open circles show the simulation results obtained through $G=p\left|\mathbf{F}^{G}\right| /$ $|\mathbf{v}|, D=\left|\mathbf{F}^{D}\right| /|\mathbf{v}|$. The solid line corresponds to the result obtained from an explicit expression of $G\left(H_{\mathrm{p}}\right)=G(0)\left[1-p\left(H_{\mathrm{p}} / H_{\mathrm{s}}\right)\right]$, as reported in Ref. 15, where $H_{\mathrm{s}}=10 \mathrm{kOe}$ was used for our nanodot dimensions.
$X=|\mathbf{X}|$, the vortex core was shifted from the initial center position and then relaxed, as shown in Fig. 1(b). The slope in the plot of $W$ versus $X^{2}$ accordingly gives rise to $\kappa / 2$, as seen in Fig. 4(a). The resultant $H_{\mathrm{p}}$-dependent $\kappa$ values are shown in Fig. 4(b) (see open circles). The value of $\kappa$ at $H_{\mathrm{p}}=0$ is estimated to $\sim 2.635 \mathrm{erg} / \mathrm{cm}^{2}$, which value is smaller by $11 \%$ than that of the analytical expression reported in Refs. 11 and 13. This seems that the analytical expression has been derived assuming the two-vortices model ${ }^{11,13,21}$ that is different from dynamic vortex configurations in the simulations. The $\kappa$ value decreases with increasing $\left|H_{\mathrm{p}}\right|$, and the parabolic curve is somewhat asymmetric at about $H_{\mathrm{p}}=0$ in contrast to the explicit parabolic expression (solid line) of $\kappa\left(H_{\mathrm{p}}\right)=\kappa(0)\left[1-\left(H_{\mathrm{p}} / H_{\mathrm{s}}\right)^{2}\right]$ reported in Ref. 15. This explicit form was obtained for the assumption that the potential energy profiles are the same for the different signs of $+H_{\mathrm{p}}$ and $-H_{\mathrm{p}}$. The asymmetry of our result originates from the difference in the $m_{\mathrm{z}}$ profile of the vortex core between $+H_{\mathrm{p}}$ and $-H_{\mathrm{p}}$, shown in the inset of Fig. 4(b). This appears to break the symmetry of $\kappa$ at around $H_{\mathrm{p}}=0$. The difference in $\omega_{0}$ between our simulation results (closed circles) and the analytical estimation (solid line) in Ref. 15 can be ascribed mainly to the difference in $\kappa: \kappa(-3 \mathrm{kOe})=2.232 \mathrm{erg} / \mathrm{cm}^{2}, \kappa(+3$ $\mathrm{kOe})=2.365 \mathrm{erg} / \mathrm{cm}^{2}$, from our simulation, and $\kappa(-3$ $\mathrm{kOe})=\kappa(+3 \mathrm{kOe})=2.591 \mathrm{erg} / \mathrm{cm}^{2}$, from the analytical equation. The core $m_{\mathrm{z}}$ profiles not considered in the analytical expression would give rise to incorrect values of $\kappa$ for positive and negative $H_{\mathrm{p}}$ values as can be evidenced by excellent agreements between the simulation results of $\omega_{0}\left(H_{\mathrm{p}}\right)$ (closed circles) and the results of $\kappa\left(H_{\mathrm{p}}\right) / G\left(H_{\mathrm{p}}\right)$ (open circles), shown in Fig. 2.

(a)

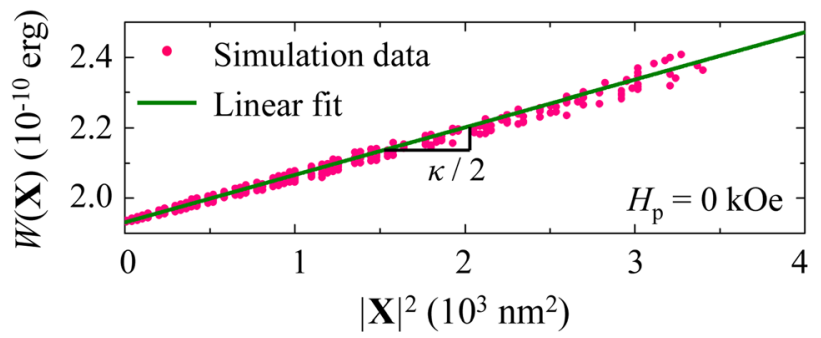

(b)

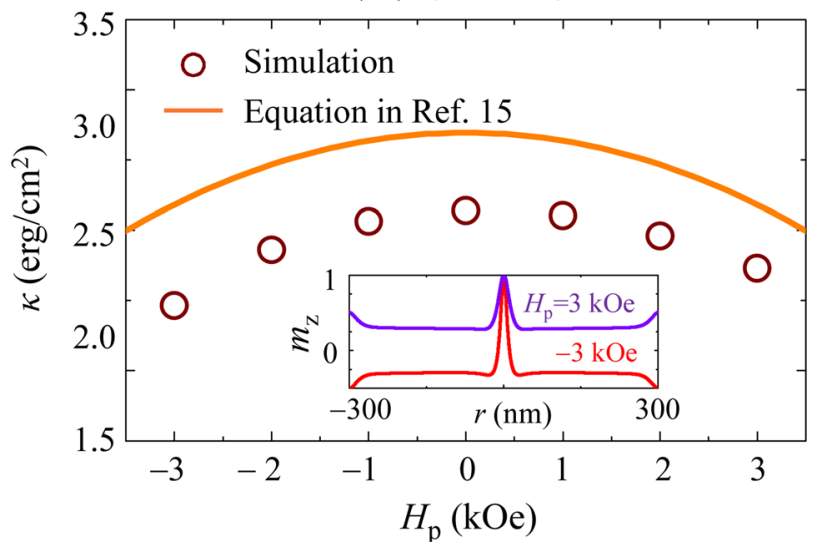

FIG. 4. (Color online) (a) Calculated total energy $W(\mathbf{X})$ vs $|\mathbf{X}|^{2}$ for $H_{\mathrm{p}}=0$ kOe. The solid line indicates a linear fit to the simulation results (dots). (b) Dependence of $\kappa$ on $H_{\mathrm{p}}$. The open circles indicate values obtained directly from the simulation results, while the solid line corresponds to the result for $\kappa\left(H_{\mathrm{p}}\right)=\kappa(0)\left[1-\left(H_{\mathrm{p}} / H_{\mathrm{s}}\right)^{2}\right]$ reported in Ref. 15. The inset shows the comparison of the core-region $m_{\mathrm{z}}$ profiles for $H_{\mathrm{p}}=+3$ and $-3 \mathrm{kOe}$. 


\section{CONCLUSIONS}

We studied, by micromagnetic simulations combined with an analytical approach, the dependence of the angular frequency of vortex core oscillations upon $H_{\mathrm{p}}$. The observed linear increase of the vortex eigenfrequency with $H_{\mathrm{p}}$ could be explained quantitatively in terms of the $H_{\mathrm{p}}$ dependences of all of the vortex dynamic variables, $G, \kappa$, and $D$, calculated numerically from the micromagnetic simulation results. This work shows how numerical simulations and their quantitative interpretations promise a more correct understanding of vortex dynamics in nanodots.

\section{ACKNOWLEDGMENTS}

This work was supported by the Basic Science Research Program through the National Research Foundation of Korea (NRF) funded by the Ministry of Education, Science and Technology (No. 20100000706).

${ }^{1}$ A. Hubert and R. Schäfer, Magnetic Domains (Springer-Verlag, Berlin, 1998).

${ }^{2}$ T. Shinjo, T. Okuno, R. Hassdorf, K. Shigeto, and T. Ono, Science 289 , 930 (2000).

${ }^{3}$ B. Van Waeyenberge, A. Puzic, H. Stoll, K. W. Chou, T. Tyliszczak, R. Hertel, M. Fähnle, H. Brückl, K. Rott, G. Reiss, I. Neudecker, D. Weiss, C. H. Back, and G. Schütz, Nature 444, 461 (2006).

${ }^{4}$ K. Yamada, S. Kasai, Y. Nakatani, K. Kobayashi, H. Kohno, A. Thiaville, and T. Ono, Nat. Mater. 6, 269 (2007); S.-K. Kim, Y.-S. Choi, K.-S. Lee, K. Y. Guslienko, and D.-E. Jeong, Appl. Phys. Lett. 91, 082506 (2007).

${ }^{5}$ S. Choi, K.-S. Lee, K. Yu. Guslienko, and S.-K. Kim, Phys. Rev. Lett. 98, 087205 (2007).

${ }^{6}$ Q. F. Xiao, J. Rudge, E. Girgis, J. Kolthammer, B. C. Choi, Y. K. Hong, and G. W. Donohoe, J. Appl. Phys. 102, 103904 (2007); R. Hertel,
S. Gliga, M. Fähnle, and C. M. Schneider, Phys. Rev. Lett. 98, 117201 (2007).

${ }^{7}$ K.-S. Lee, K. Yu. Guslienko, J.-Y. Lee, and S.-K. Kim, Phys. Rev. B 76, 174410 (2007);K. Y. Guslienko, K.-S. Lee, and S.-K. Kim, Phys. Rev. Lett. 100, 027203 (2008).

${ }^{8}$ R. P. Cowburn, Nat. Mater. 6, 255 (2007); J. Thomas, Nature Nanotech. 2, 206 (2007).

${ }^{9}$ Y.-S. Choi, M.-W. Yoo, K. -S. Lee, Y.-S. Yu, H. Jung, and S.-K. Kim, Appl. Phys. Lett. 96, 072507 (2010).

${ }^{10}$ S.-K. Kim, K.-S. Lee, Y.-S. Yu, and Y.-S. Choi, Appl. Phys. Lett. 92, 022509 (2008).

${ }^{11}$ K. Yu. Guslienko, B. A. Ivanov, V. Novosad, H. Shima, Y. Otani, and K. Fukamichi, J. Appl. Phys. 91, 8037 (2002).

${ }^{12}$ A. Dussaux, B. Georges, J. Grollier, V. Cros, A. V. Khvalkovskiy, A. Fukushima, M. Konoto, H. Kubota, K. Yakushiji, S. Yuasa, K. A. Zvezdin, K. Ando, and A. Fert, Nat. Commun. 1, 8 (2010).

${ }^{13}$ K. Yu Guslienko, X. F. Han, D. J. Keavney, R. Divan, and S. D. Bader, Phys. Rev. Lett. 96, 067205 (2006).

${ }^{14}$ Y.-S. Choi, S.-K. Kim, K.-S. Lee, and Y.-S. Yu, Appl. Phys. Lett. 93, 182508 (2008); Y.-S. Choi, K.-S. Lee, and S.-K. Kim, Phys. Rev. B 79, 184424 (2009).

${ }^{15}$ G. de Loubens, A. Riegler, B. Pigeau, F. Lochner, F. Boust, K. Y. Guslienko, H. Hurdequint, L. W. Molekamp, G. Schmidt, A. N. Slavin, V. S. Tiberkevich, N. Vukadinovic, and O. Klein, Phys. Rev. Lett. 102, 177602 (2009).

${ }^{16}$ A. A. Thiele, Phys. Rev. Lett. 30, 230 (1973); D. L. Huber, Phys. Rev. B 26, 3758 (1982).

${ }^{17} \mathrm{We}$ used the $1.2 \mathrm{a} 4$ version of the OOMMF code.See http://math.nist.gov/ oommf.

${ }^{18}$ L. D. Landau and E. M. Lifshitz, Phys. Z. Sowjetunion 8, 153 (1935); T. L. Gilbert, Phys. Rev. 100, 1243 (1955).

${ }^{19}$ At fields smaller than $\mathrm{Hp}=-3 \mathrm{kOe}$, it is too difficult to investigate vortexcore reversals as they are very unstable.

${ }^{20}$ K.-S. Lee and S.-K. Kim, Phys. Rev. B 78, 014405 (2008).

${ }^{21}$ K. L. Metlov and K. Y. Guslienko, J. Magn. Magn. Mater. 242, 1015 (2002).

${ }^{22}$ K. Yu. Guslienko, Appl. Phys. Lett. 89, 022510 (2006).

${ }^{23}$ N. A. Usov, and S. E. Peschany J. Magn. Magn. Mater. 118, L290 (1993). 